ORIGINAL ARTICLE

\title{
Altered structural and effective connectivity in anorexia and bulimia nervosa in circuits that regulate energy and reward homeostasis
}

\author{
GKW Frank ${ }^{1,2}$, ME Shott ${ }^{1}$, J Riederer $^{1}$ and TL Pryor $^{3}$
}

\begin{abstract}
Anorexia and bulimia nervosa are severe eating disorders that share many behaviors. Structural and functional brain circuits could provide biological links that those disorders have in common. We recruited 77 young adult women, 26 healthy controls, 26 women with anorexia and 25 women with bulimia nervosa. Probabilistic tractography was used to map white matter connectivity strength across taste and food intake regulating brain circuits. An independent multisample greedy equivalence search algorithm tested effective connectivity between those regions during sucrose tasting. Anorexia and bulimia nervosa had greater structural connectivity in pathways between insula, orbitofrontal cortex and ventral striatum, but lower connectivity from orbitofrontal cortex and amygdala to the hypothalamus $(P<0.05$, corrected for comorbidity, medication and multiple comparisons). Functionally, in controls the hypothalamus drove ventral striatal activity, but in anorexia and bulimia nervosa effective connectivity was directed from anterior cingulate via ventral striatum to the hypothalamus. Across all groups, sweetness perception was predicted by connectivity strength in pathways connecting to the middle orbitofrontal cortex. This study provides evidence that white matter structural as well as effective connectivity within the energy-homeostasis and food reward-regulating circuitry is fundamentally different in anorexia and bulimia nervosa compared with that in controls. In eating disorders, anterior cingulate cognitiveemotional top down control could affect food reward and eating drive, override hypothalamic inputs to the ventral striatum and enable prolonged food restriction.
\end{abstract}

Translational Psychiatry (2016) 6, e932; doi:10.1038/tp.2016.199; published online 1 November 2016

\section{INTRODUCTION}

Anorexia and bulimia nervosa are severe psychiatric disorders with high mortality. ${ }^{1}$ Although anorexia nervosa is mainly characterized by severe underweight and bulimia nervosa individuals are at normal to high weight and regularly binge and purge, ${ }^{2}$ there are many overlapping symptoms, such as food restriction, excessive exercise, altered interoceptive perception including hunger and appetite $^{3}$ as well drive for thinness and body dissatisfaction. Anorexia and bulimia nervosa aggregate in families, and shared biological underpinnings have been hypothesized. ${ }^{4}$ The brain circuitry of food intake regulation depends on the interaction of the hypothalamus, which has a central role in energy homeostasis, with brain regions such as prefrontal and orbitofrontal cortex (OFC), insula, midbrain and ventral striatum, a brain circuitry that integrates taste perception, food reward value and cognitiveemotional associations with food..$^{5}$ Alterations in those circuits in both disorders could point toward shared vulnerabilities.

Brain research in eating disorders has started to shed light on how altered brain structure or function may be common to anorexia and bulimia nervosa. Positron emission tomography studies suggested in both disorders increased serotonin $1 \mathrm{~A}$ as well as cannabinoid type 1 receptors, ${ }^{6,7}$ neurotransmitter receptors associated with food intake modulation and sweet taste perception. ${ }^{8-10}$ Structural brain imaging studies have been inconsistent. One study that directly compared the two disorders in a nutritionally highly controlled environment found larger OFC volumes in anorexia and bulimia nervosa ${ }^{11}$ - a brain region that processes food pleasantness and regulates food intake. ${ }^{12}$ Others found increased somatosensory cortex volumes in both disorders. ${ }^{13}$ Functional magnetic resonance brain imaging (fMRI) indicated greater insula activation to food images, ${ }^{14}$ as well as higher resting-state synchrony between anterior cingulate cortex and precuneus, ${ }^{15}$ although other studies found opposite brain activation in response to visual food cues ${ }^{16}$ or sweet taste stimulation. ${ }^{17-19}$ Studies that focused on brain white matter found reduced white matter integrity (fractional anisotropy, FA) in anorexia and bulimia nervosa within the fornix. ${ }^{20-22}$

Research has helped to better understand how brain networks are structurally or functionally connected. ${ }^{23}$ One method is to investigate how strongly white matter tracts connect those brain regions, expressed as streamlines as an indicator of fiber amount. ${ }^{24}$ Another method is to investigate how brain regions functionally interact; this so-called dynamic causal or effective connectivity provides indication that brain region drives activation in another. ${ }^{25,26} \mathrm{~A}$ few studies exist in eating disorders that have investigated effective connectivity, but not with respect to taste processing, which is the focus of our work. One study using resting-state data and Granger causality found that individuals

${ }^{1}$ Department of Psychiatry, University of Colorado School of Medicine, University of Colorado Anschutz Medical Campus, Aurora, CO, USA; ${ }^{2}$ Neuroscience Program, University of Colorado Denver, Anschutz Medical Campus, Aurora, CO, USA and ${ }^{3}$ Eating Disorders Center Denver, Denver, CO, USA. Correspondence: Dr GKW Frank, Departments of Psychiatry and Neuroscience, Developmental Brain Research Program, University of Colorado Anschutz Medical Campus, Children's Hospital Colorado, Gary Pavilion A036/B-130, 13123 East 16th Avenue, Aurora, CO 80045, USA.

E-mail: Guido.Frank@ucdenver.edu

Received 14 January 2016; revised 18 August 2016; accepted 24 August 2016 
with anorexia nervosa had higher effective connectivity from medial OFC and insula to inferior frontal gyrus, but lower effective connectivity from the frontal gyrus to the cingulate cortex. ${ }^{27}$ One small study suggested connectivity differences between anorexia nervosa and control groups between medial occipital cortex, extrastriate and fusiform body areas in response to viewing pictures of bodies or chairs. ${ }^{28} \mathrm{~A}$ study where individuals with anorexia, bulimia nervosa and controls viewed images of food and non-food items found no effective connectivity differences between insula, orbitofrontal and frontal cortex between anorexia nervosa and controls, but the bulimia nervosa group did not have connectivity between left insula and right frontal cortex that the other study groups had. ${ }^{14} \mathrm{~A}$ very recent report studied connectivity between the nucleus accumbens and the OFC using resting-state images in anorexia nervosa at two time points, before and after weight restoration. ${ }^{29}$ There the anorexia nervosa group showed an effective connectivity direction from the OFC to the nucleus accumbens, as well as greater anatomical connectivity strength using probabilistic tractography between the two regions of interest. One study from our group in individuals long-term recovered from anorexia nervosa found greater white matter connectivity strength from the insula to ventral striatum and OFC. $^{30}$ Interestingly, duration of illness positively predicted connectivity strength of those tracts, suggesting a process compensating for effects from the illness and maybe affecting food reward-circuit function. In summary, few studies are available on effective or structural connectivity in eating disorders, and the varying methods and results do not provide a uniform model of brain function. One question that has to be raised in this context is what type of task is most suitable to elicit effective connectivity. We chose taste processing because we have a good understanding of the taste and taste-reward circuitry in the brain. Here we wanted to study this circuitry across multiple regions for a comprehensive assessment of structural and effective connectivity.

In this study we used a multimodal imaging approach to test the hypothesis that individuals ill with anorexia and bulimia nervosa have greater white matter connectivity across the energy homeostasis and cognitive-emotional reward circuitry. Furthermore, we wanted to study how regional activation during taste of sucrose solution is coupled, that is, what the pattern of effective connectivity or neural information flow is within this circuitry. We expected that we would find in the eating-disorder groups' indication that cognitive control regions would influence subcortical appetite and taste reward function, suggesting a topdown control mechanism. ${ }^{31}$ In contrast, we expected that in controls hypothalamic signals would rather influence reward system activation, presumably transmitting energy balance information to drive the food approach. ${ }^{32}$

\section{MATERIALS AND METHODS}

\section{Participants}

Twenty-six women with restricting-type anorexia nervosa, 25 with bulimia nervosa, as well as 26 healthy comparison women participated in the study. The sample size was based on our previous study that indicated adequate power for this type of study and analysis. ${ }^{30}$ Participants in the eating-disorder groups were recruited from Children's Hospital Colorado or Eating Disorders Center Denver. Eating disorder subjects were within 1-2 weeks of program-prescribed food intake to avoid acute effects of starvation and dehydration. Healthy comparison women were recruited through local advertisements. The Structured Clinical Interview for Diagnostic and Statistical Manual of Mental Disorders, 4th Edition diagnoses was administered by a doctoral-level interviewer. All participants were right-handed, without history of head trauma, neurological disease, major medical illness, psychosis or substance-use disorders. The study was approved by the Colorado Multiple Institutional Review Board, and all participants provided written informed consent.

\section{Behavior assessments}

Study participants completed the Eating Disorder Inventory-3 (EDI-3), ${ }^{33}$ Temperament and Character Inventory (TCI), ${ }^{34}$ Spielberger State and Trait Anxiety Inventory (STAI), ${ }^{35}$ Beck Depression Inventory (BDI) ${ }^{36}$ and Revised Sensitivity to Punishment and Reward Questionnaire (SPSRQ). ${ }^{37}$ Before brain-imaging subjects rated sucrose sweetness on a 9-point likert scale, $1=$ dislike very much to $9=$ like very much.

\section{Brain imaging procedures}

Before brain imaging between 0800 and 0900 hours, eating-disorder individuals ate their meal plan breakfast; controls had a breakfast matched in quality and calories to the average meal plan breakfast. Brain images were acquired on a GE Signa 3T scanner: (1) diffusion-weighted imaging (DWI) included 25 DWI diffusion directions and one T2-weighted $(b=0)$ baseline image; 45 slices per image in anterior-posterior commissure orientation $(128 \times 128$ matrix, repetition time $(T R) /$ echo time $(T E)=16000-$ $/ 82.6 \mathrm{~ms}$, field of view $=26 \mathrm{~cm}, b$-value $=1000$, ASSET, slice thickness $/$ gap $=2.6 / 0 \mathrm{~mm}$ ). (2) fMRI T2* weighted echo-planar imaging for blood oxygen-dependent functional activity was performed, voxel size $3.4 \times 3.4 \times 2.6 \mathrm{~mm}$, TR $2100 \mathrm{~ms}$, TE $30 \mathrm{~ms}$, angle $70^{\circ}, 30$ slices, interleaved acquisition and $2.6 \mathrm{~mm}$ slice thickness with $1.4 \mathrm{~mm}$ gap.

\section{fMRI task}

We adapted the design used by O'Doherty et al..$^{38}$ Individuals received three taste stimuli during fMRI imaging: $1 \mathrm{~mol} \mathrm{I}^{-1}$ sucrose solution (100 trials), no solution (100 trials) and artificial saliva (80 trials). Individuals learned to associate each unconditioned taste stimulus (US) with a paired conditioned visual stimulus (CS) that is probabilistically associated with its US: the CS shape for sucrose was followed in $80 \%$ of trials by sucrose solution (the other $20 \%$ were followed by no solution), and the CS shape associated with no-solution (null) was followed in $80 \%$ of the trials by no solution (the other $20 \%$ were followed by sucrose); the CS shape for artificial saliva was always followed by saliva receipt. For each subject, the first 10 trials were fixed CS shape for sucrose followed by the delivery of US sucrose to establish an initial stable association between the CS sucrose shape and US sucrose taste. ${ }^{38}$ All other trials were fully randomized without predetermined order. The taste stimuli were applied using a customized-programmable syringe pump (J-Kem Scientific, St Louis, MO, USA) controlled with the E-Prime Software (Psychological Software Tools, Pittsburgh, PA, USA). Individual taste application was triggered by magnetic resonance imaging scanner radiofrequency pulse. ${ }^{18}$ Task duration was $28 \mathrm{~min}$.

\section{Diffusion image analysis}

Diffusion weighted images were processed using FSL's Diffusion Toolbox 4.1.3 (FDT, Oxford Centre for Functional MRI of the Brain, http://www.fmrib. ox.ac.uk/fsl). Images were corrected for eddy current distortions and head motion. Probabilistic fiber tractography was computed for each subject using PROBTRACKX2 to generate the most probable connectivity distribution between seed and ipsilateral target. Tractography parameters were as follows: 5000 sample tracts per seed voxel, 0.2 curvature threshold, step length of 0.5 and a maximum number of steps 2000 . Connectivity was assessed by computing connection strength that determines the mean probability of streamlines for each seed-target combination. The calculated connection strength value was divided by the total connection probability of seed regions and then multiplied by the mean connection probability across seed and target regions and finally divided by the target volume of interest in order to normalize and rescale the results for size of seed and target regions. ${ }^{39}$ Physical path length was also corrected for. ${ }^{39}$ In each hemisphere, tract-based connection strength was calculated for anatomical white matter tracts connecting regions of a comprehensive taste reward hierarchy proposed by Rolls et al. ${ }^{40}$ (Supplementary Figure 1). Seed regions included the thalamus, dorsal anterior insula, ventral anterior insula, posterior insula, substantia nigra, central nucleus of the amygdala, basolateral amygdala, medial OFC, middle OFC, gyrus rectus and inferior OFC. Thalamus targets included all subregions of the insula and the frontal operculum. Targets of insula subregions included the basolateral amygdala, central nucleus of the amygdala, ventral striatum, medial prefrontal cortex (PFC), medial OFC, middle OFC, gyrus rectus and the inferior OFC. The ventral striatum was the target of the substantia nigra seed region. For both the central and the basolateral nucleus of the amygdala, the targets were the hypothalamus, substantia nigra, ventral 
striatum and the anterior cingulate cortex. OFC subregion targets included the hypothalamus, ventral striatum and the medial PFC. In total, tractbased connection strength was calculated for 98 white matter tracts connecting aforementioned seed and ipsilateral targets. The Automated Anatomical Labeling atlas was used to determine coordinates for each seed and target region. ${ }^{41}$

\section{fMRI analysis}

Brain-imaging data were preprocessed and analyzed using the SPM8 software in Matlab R2009b, 7.9.0 (MathWorks, Natick, MA, USA). Data from each subject were realigned to the first volume, normalized to the Montreal Neurological Institute template and smoothed with a 6-mm full width at half maximum Gaussian kernel. Each image sequence was manually inspected, and images with artifacts or movement more than one voxel size were removed.

Data were modeled with a hemodynamic response function-convolved boxcar function-using the general linear model, including temporal and dispersion derivatives, and autoregression. A 128-s highpass filter was applied to remove low-frequency fluctuation in the BOLD signal. Motion parameters were applied as regressors in the first-level analysis to correct for individual movement. We then developed first-level models in which we predicted the response in each voxel as a function of each of the stimulus conditions. For this study we computed the contrast for expected sucrose receipt versus expected receipt of no solution.

\section{Effective connectivity}

Within SPM8, we extracted functional time-series data for expected receipt of $1 \mathrm{~m}$ sucrose solution for each of the seed and target regions of interest using the SPM marsbar toolbox. Effective connectivity was inferred using Independent Multiplesample Greedy Equivalence Search (IMaGES) and Linear non-gaussian Orientation, Fixed Structure search algorithms housed within the TETRAD V program. ${ }^{42}$ The goal of effective connectivity analyses is to understand causal relations among the neuronal populations whose activity gives rise to observed fMRI signals in spatially localized regions of interest. The results from those analyses are presented as directed graphs, where nodes or vertices in the graph represent brain regions and directed edges in the graph represent relatively direct causal influences of one region on another. The Independent Multiplesample Greedy Equivalence Search (IMaGES) is a modification of the Greedy Equivalence Search (GES) that allows to analyze multiple data sets. GES begins with an empty graph whose vertices are the recorded variables and proceeds to search forward, one new connection at a time, over Markov Equivalence classes of directed acyclic graphs. Each class of models with an additional edge is scored using the Bayes Information Criterion: $-2 \ln (\mathrm{ML})+k \ln (n)$, where $\mathrm{ML}$ is the maximum likelihood estimate, $k$ is the dimension of the model (the number of directed edges plus the number of variables) and $n$ is the sample size. The algorithm searches forward from the empty graph until no improvement in the Bayes Information Criterion score is possible, and then backward, and outputs a description of a Markov Equivalence class. In practice, the algorithm requires a computation of a series of maximum likelihood estimates, and is limited to cases where approximations to such estimates can be rapidly obtained. The analysis process in IMaGES and GES is nonlinear, and therefore a comparison of a parameterized output of the GES using conventional linear models for group comparison is not recommended. IMaGES was supplemented by a Linear non-gaussian Orientation, Fixed Structure algorithm postprocessor; this leads to a precision of orientations that is greater than $90 \%$ and the precision of recall greater than $80 \%$, that is, more edges are directed than with IMaGES alone, and with no loss of accuracy. ${ }^{25}$

\section{Statistical analyses}

Demographic and behavioral data were analyzed using SPSS 23.0 (IBMSPSS, Chicago, IL, USA) using multivariate multivariate analyses of variance (MANOVAs); extracted data for brain connectivity were in addition corrected for medication use, anxiety and mood disorder diagnoses (added factors in the model) and post hoc pairwise between group analyses were Bonferroni-corrected. In addition, we analyzed the connectivity data with MANOVA without added factors as well as a nonparametric Kruskal-Wallis test because not all data in each group were normally distributed (Supplementary Table 2). Linear regression analyses to test behavior-brain relationships were applied for age, body mass index and $1 \mathrm{~m}$ sucrose ratings for pleasantness and sweetness; additional exploratory analyses tested the potentially confounding effects of depression or anxiety measures. Significant correlations were corrected using the false discovery rate using the method proposed by Benjamini and Hochberg. ${ }^{43}$

\section{RESULTS}

Demographics and assessment results

Groups were matched for age; anorexia nervosa subjects had significantly (Table 1) lower body mass index compared with the other study groups; harm avoidance, depression, drive for thinness, body dissatisfaction, state and trait anxiety and sensitivity to punishment were higher in both eating-disorder groups compared with controls. Sensitivity to reward was greater compared with controls only in the bulimia nervosa group, whereas noveltyseeking was lower in the anorexia nervosa group.

\section{White matter connectivity strength}

Left: white matter connection strength (Figure 1 and Supplementary Table 1) was higher in both eating disorder groups between insula regions and middle OFC, between ventral and dorsal anterior insula to ventral striatum and dorsal anterior insula, from posterior insula to medial PFC, and between inferior OFC and gyrus rectus and ventral striatum. In anorexia and bulimia nervosa groups, connectivity strength was lower between middle OFC and hypothalamus, as well as between gyrus rectus and medial PFC. In bulimia nervosa only, connectivity was lower compared with controls from ventral anterior insula to inferior OFC and central nucleus of the amygdala, and in anorexia nervosa between medial OFC and hypothalamus.

Right: Connectivity strength was greater in both eating-disorder groups in tracts from posterior insula and medial OFC to ventral striatum and from dorsal anterior insula to the medial PFC.

Anorexia and bulimia nervosa groups showed less connectivity between basolateral nucleus of the amygdala and hypothalamus; anorexia nervosa had less connectivity between middle OFC and hypothalamus, and bulimia nervosa from the amygdala basolateral nucleus to the ventral striatum and dorsal anterior insula.

The additional analysis using MANOVA without comorbidity or medication as factors, or the analysis using the non-parametric test indicated that the MANOVA with added factors did not inflate results (Supplementary Table 1).

\section{Effective dynamic connectivity}

There were bilateral effective connectivity patterns that all groups (Figure 2 and Table 2) shared: on the left from insula regions to the thalamus and ventral striatum, and on the right from the ventral anterior insula to OFC as well as intra-insular and orbitofrontal connections.

Only the controls had an effective connectivity pattern from the hypothalamus to ventral striatum bilaterally. On the right side both eating-disorder groups showed effective connectivity from the anterior cingulate to ventral striatum, and from there to the hypothalamus. Substantia nigra effectively connected to the thalamus, whereas the opposite relationship was the case in controls.

Unique to anorexia nervosa was left- and right-sided connectivity driven by the frontal operculum to the anterior cingulate cortex; the bulimia nervosa group showed a unique pattern of dynamic connectivity from anterior cingulate to medial OFC. Both anorexia and bulimia nervosa showed effective connectivity on the left from ventral anterior insula to inferior OFC, middle to inferior OFC and dorsal to ventral anterior insula.

\section{Correlation analyses}

Age, body mass index or pleasantness perception were not significantly correlated with brain results in any group or pathway 
Table 1. Demographic and behavioral data

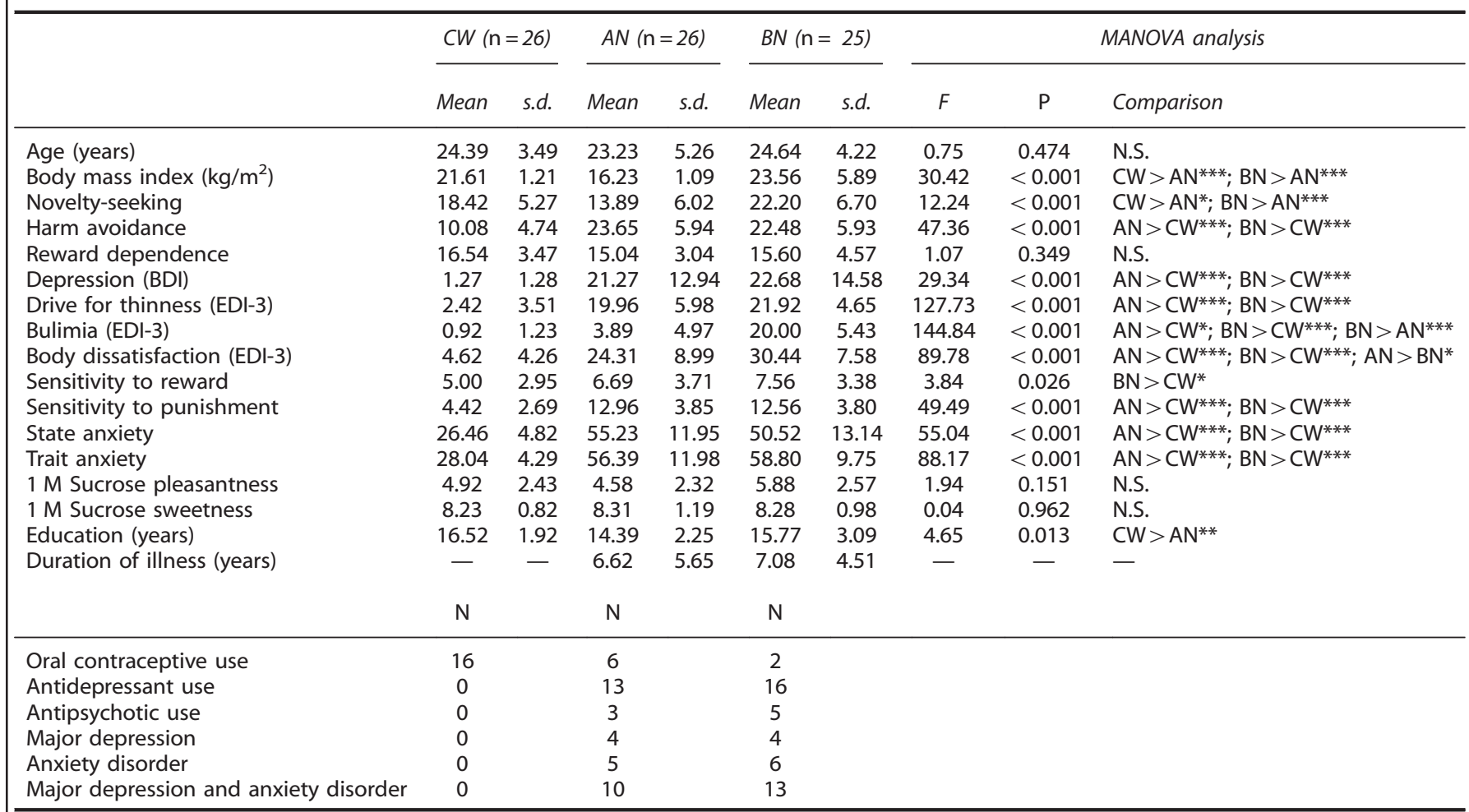

Abbreviations: AN, anorexia nervosa; BDI, Beck Depression Inventory; BN, bulimia nervosa; CW, control women; EDI-3, Eating Disorder Inventory-3; MANOVA, multivariate analysis of variance; N.S., non significant. ${ }^{*} P<0.05,{ }^{* *} P<0.01,{ }^{* * *} P<0.001$. Significance is based on the Dunnett's T3 post hoc test.

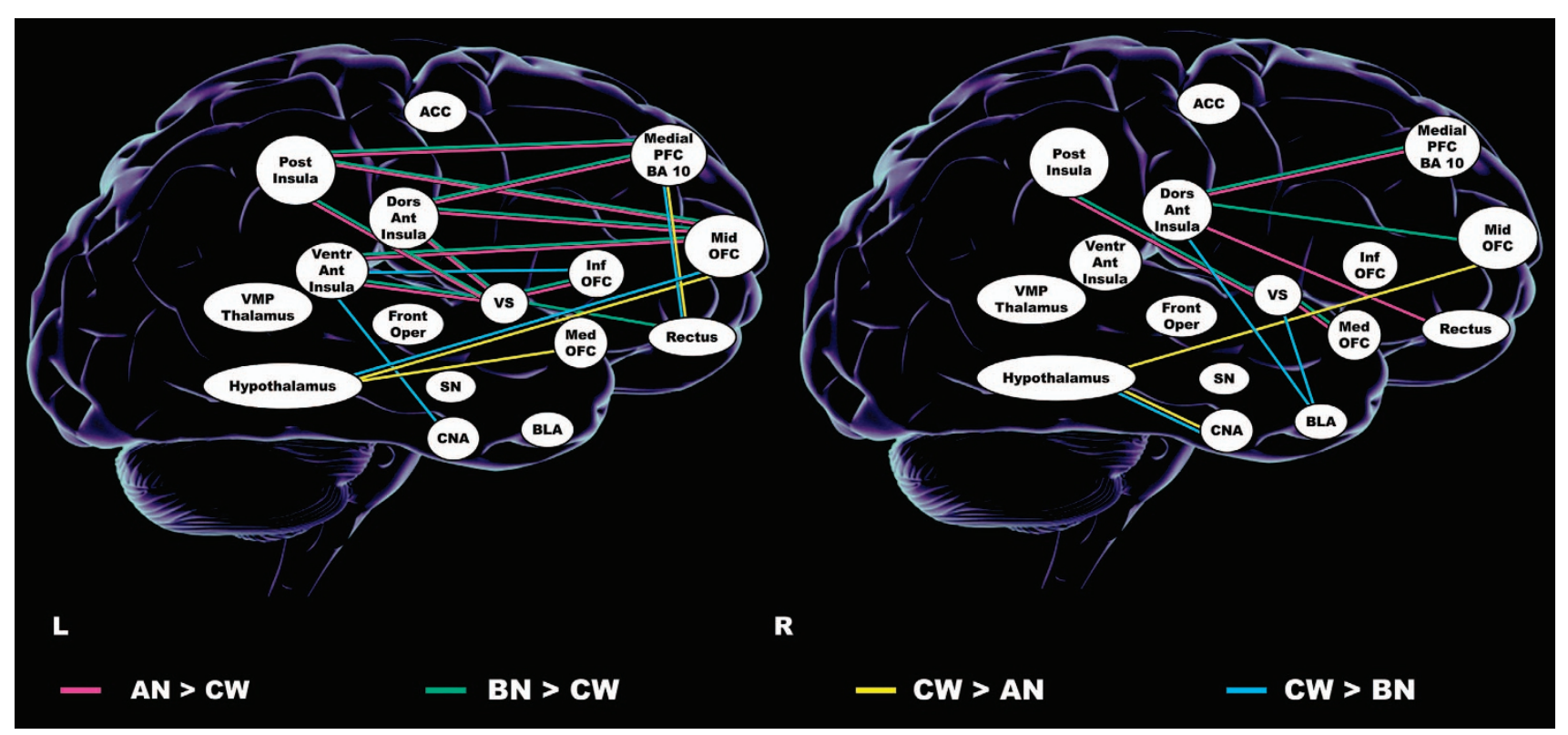

Figure 1. Connection strength results. ACC, anterior cingulate cortex; AN, anorexia nervosa; BLA, basolateral amygdala; BN, bulimia nervosa; CNA, central nucleus of the amygdala; CW, Controls; Dors Ant Insula, dorsal anterior insula; Front Oper, frontal operculum; Inf OFC, inferior orbitofrontal cortex; L, left; Med OFC, medial orbitofrontal Cortex; Medial PFC, BA 10, medial prefrontal cortex, Brodmann Area 10; Mid OFC, middle orbitofrontal cortex; Post Insula, posterior insula; R, right; Rectus, gyrus rectus; SN, substantia nigra; Ventr Ant Insula, ventral anterior insula; VS, ventral striatum; VMP Thalamus, ventral posterior medial thalamus.

after false discovery rate correction. All groups showed positive correlations between sweetness perception and connection strength in fibers that terminated in the middle OFC, although right hemispheric in controls and left-sided in eating-disorder groups (Table 3). In addition, in the anorexia nervosa group only, there was a pattern of negative correlation for pathways between thalamus, hypothalamus, insula and limbic brain regions. Duration of illness was positively correlated with connection strength for 

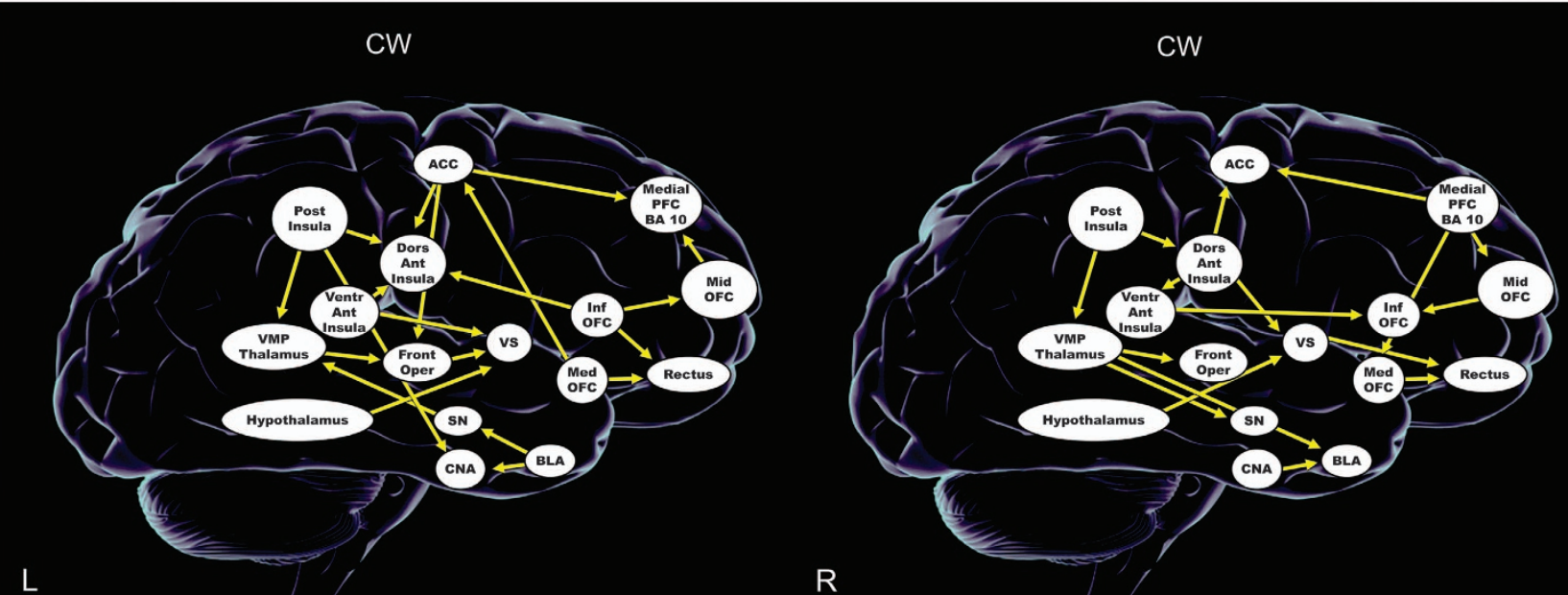

AN

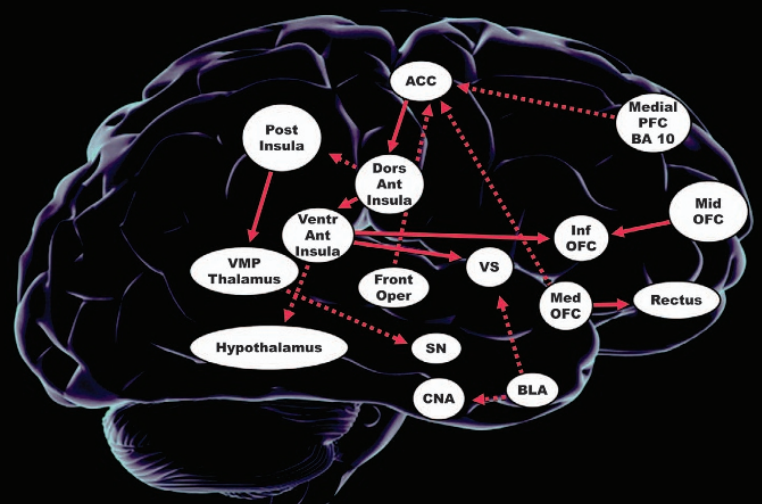

L

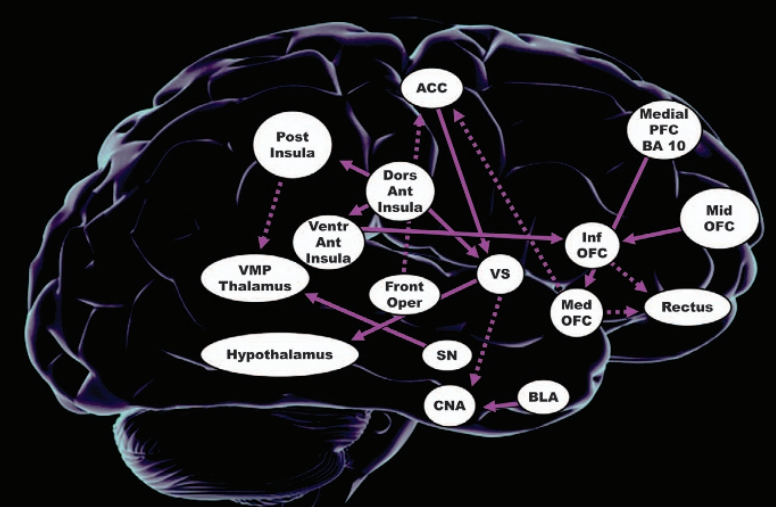

R

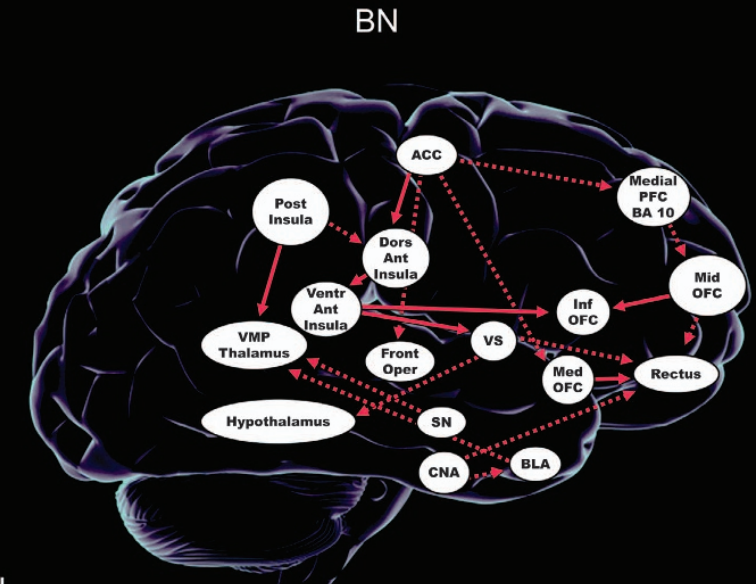

L

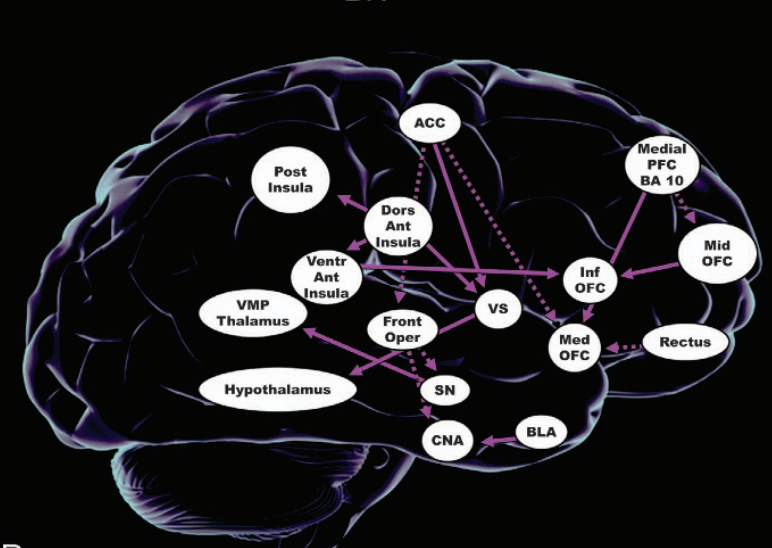

$\mathrm{R}$

Figure 2. Effective connectivity. ACC, anterior cingulate cortex; AN, anorexia nervosa; BLA, basolateral amygdala; BN, bulimia nervosa; CNA, central nucleus of the amygdala; CW, Controls; Dors Ant Insula, dorsal anterior insula; Front Oper, frontal operculum; Inf OFC, inferior orbitofrontal cortex; L, left; Med OFC, medial orbitofrontal cortex; Medial PFC, BA 10, medial prefrontal cortex, Brodmann Area 10; Mid OFC, middle orbitofrontal cortex; Post Insula, posterior insula; R, right; Rectus, gyrus rectus; SN, substantia nigra; Ventr Ant Insula, ventral anterior insula; VMP Thalamus, ventral posterior medial thalamus; VS, ventral striatum. For AN and BN, solid lines indicate similar pattern and dashed lines indicate different pattern between AN and BN groups. Yellow lines are used for left- and right-sided connections in the CW. For the AN and BN groups, red lines indicate left-sided connections and purple lines indicate right-sided connections. 
Table 2. Effective connectivity results

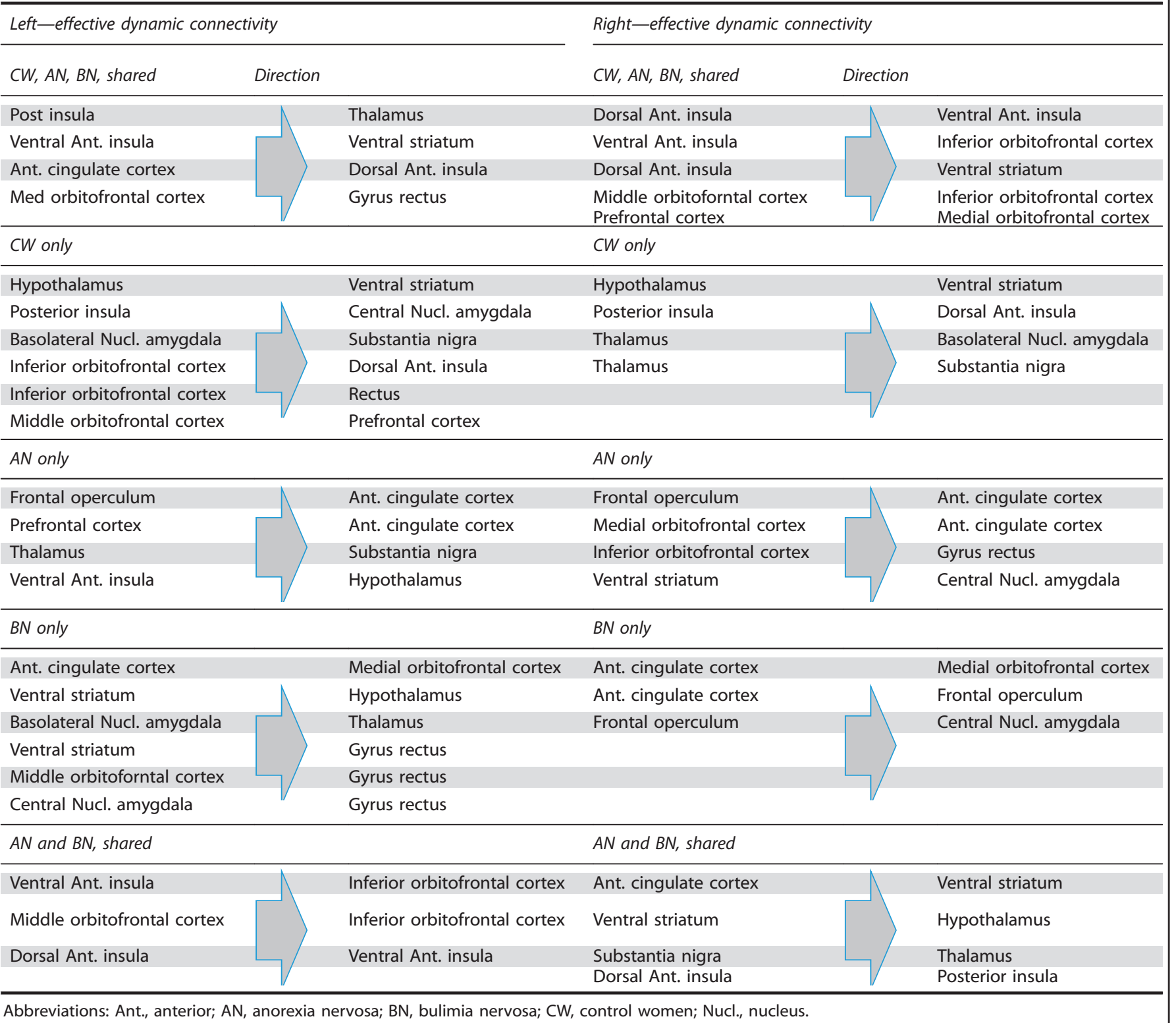

Abbreviations: Ant., anterior; AN, anorexia nervosa; BN, bulimia nervosa; CW, control women; Nucl., nucleus.

five pathways in the eating-disorder groups, but none survived false discovery rate correction.

To test for confounding variables, we tested whether group differences in regional brain response to sucrose solution was related to connection strength or effective connectivity. We conducted an additional whole-brain contrast for expected sucrose against no solution receipt. This analysis did not show any group differences ( $P<0.001,10$ voxel cluster threshold). In addition, we calculated FA for investigated fiber paths using fslstats, ${ }^{30}$ and tested whether FA predicted group differences for connectivity strength or effective connectivity. FA was lower $(P<0.05$, corrected for multiple comparisons, comorbidity and medication) in both anorexia and bulimia groups compared with controls from left ventral anterior insula and gyrus rectus to ventral striatum, left posterior insula to middle OFC and right middle OFC to hypothalamus. In anorexia nervosa only, FA was lower in connections from right central nucleus of amygdala to hypothalamus, left dorsal anterior insula to ventral striatum, right dorsal anterior insula to gyrus rectus, bilateral posterior insula to ventral striatum, left medial OFC to hypothalamus, right medial OFC to ventral striatum and left gyrus rectus to PFC. There were no significant correlations between FA and structural connectivity, and effective connectivity was not selectively altered in pathways with lower FA.

\section{DISCUSSION}

The results of this study indicate that both anorexia and bulimia nervosa are associated with widespread alterations in white matter structural as well as effective connectivity in taste-reward and appetite-regulating pathways. Structural connectivity was greater in both eating-disorder groups between insula and orbitoand PFC regions, whereas connection strength was lower in pathways to the hypothalamus, a region central to feeding regulation. Effective connectivity during sweet taste stimulation differed between groups, including the anterior cingulate showing effective connectivity to the ventral striatum, which modulated 
Table 3. Correlation between connection strength and $1 \mathrm{~m}$ sucrose sweetness ratings

\begin{tabular}{|c|c|c|c|c|}
\hline \multicolumn{3}{|c|}{ Correlation sweetness rating - connection strength } & $\frac{r}{0.531}$ & $\begin{array}{l}P \\
0.021\end{array}$ \\
\hline & $R$ ventral anterior insula & Middle OFC & 0.501 & 0.037 \\
\hline \multirow{7}{*}{ AN } & $\mathrm{L}$ dorsal anterior insula & Middle OFC & 0.586 & 0.038 \\
\hline & L central nucleus amygdala & ACC & -0.766 & $<0.001$ \\
\hline & L central nucleus amygdala & Hypothalamus & -0.774 & $<0.001$ \\
\hline & L posterior insula & OFC gyrus rectus & -0.605 & 0.024 \\
\hline & L substantia nigra & Ventral striatum & -0.764 & $<0.001$ \\
\hline & L ventral medial posterior nucleus & Ventral anterior insula & -0.637 & 0.011 \\
\hline & $R$ ventral medial posterior nucleus & Dorsal anterior insula & -0.650 & 0.007 \\
\hline \multirow[t]{2}{*}{$\mathrm{BN}$} & $\mathrm{L}$ dorsal anterior insula & Middle OFC & 0.534 & 0.042 \\
\hline & $\mathrm{L}$ ventral anterior insula & Middle OFC & 0.572 & 0.020 \\
\hline
\end{tabular}

Abbreviations: $A C C$, anterior cingulate cortex; $A N$, anorexia nervosa; $B N$, bulimia nervosa; $C W$, control women; FDR, false discovery rate; $L$, left; OFC, orbitofrontal cortex; R, right. All presented $P$ values are after FDR correction.

hypothalamus activity in anorexia and bulimia nervosa, whereas in controls the hypothalamus was driving ventral striatal activity. White matter structural connectivity was positively correlated with sweet taste perception in all groups in pathways that terminated in the middle OFC, but anorexia nervosa showed an additional negative correlation with connectivity strength between thalamus, hypothalamus and insula.

Human brain imaging research in anorexia and bulimia nervosa has made progress over the past years and has repeatedly implicated brain taste-reward and salience-processing regions in the pathophysiology of those disorders. Whether there are circuitry differences in structural white matter fiber connectivity and thus organization, or whether the effective functional interactions of those brain regions differ in eating disorders compared with controls had not been studied. Here we wanted to test whether greater structural white matter connectivity is a common marker of white matter organization across taste- and appetite-regulating pathways in eating disorders. Our results now indicate that anorexia and bulimia nervosa during the ill state are associated with bilaterally higher connectivity between insula, frontal cortex and ventral striatum, but on the contrary with lower connectivity between OFC and hypothalamus on the left, and lower connectivity between basolateral nucleus of the amygdala and hypothalamus on the right; anorexia nervosa showed lower connectivity between OFC and hypothalamus pathways bilaterally. Those results suggest that taste-reward circuitry alterations in eating disorder go beyond connections between insula, OFC and ventral striatum and include the hypothalamus, a key structure in appetite control. ${ }^{44}$ Animal studies have shown that the connections from the cortex and amygdala to the hypothalamus are important for cue-mediated food intake, or how much an individual eats after presentation of feeding-associated stimuli. ${ }^{45}$ Research on learning, operant conditioning and food avoidance in eating disorders is sparse. Research has shown that humans are 'innately' programmed to like sweet tastes at birth. ${ }^{46,47}$ Individuals with eating disorders typically start to avoid, for instance, eating sweets because they are afraid of gaining weight. One could see such avoidance as a form of learned behavior, and more specifically operant conditioning, with weight gain as the feared 'punishment'. ${ }^{48}$ Thus, altered functioning in frontohypothalamic circuits could facilitate or inhibit operant conditioning or reversal of such associations. Psychotherapy for meal support and nutritional rehabilitation is designed to re-establish normal eating patterns and tolerate the feared stimulus, food. However, whether those processes indeed follow the principles of operant conditioning has been insufficiently studied and deserves further exploration. The reward-system crosstalk between hypothalamus, striatum and amygdala involves neurotransmitters such as dopamine, gamma-amino-butyric acid, glutamate and orexin, ${ }^{49}$ and animal models and neurotransmitter receptor studies will be needed to further understand neurochemical alterations that could alter neural transmission.

The combination of greater connectivity and lower FA is striking. We have found a similar phenomenon in women recovered from anorexia nervosa previously. ${ }^{30} \mathrm{FA}$ is the scalar composite of axial and radial diffusivity, giving information on water diffusion across the various directions along paths. The connectivity on the other hand is a probability measure for how many fibers may connect a seed with a target region, without emphasis on radial diffusivity. Thus, the connectivity is a reflection of the number of connections that go from one point to another, whereas the FA value is thought to represent the structural integrity of those fibers. Previously, higher connectivity has been described in, for instance, in dementia of the Alzheimer's type, a condition that is by the same time associated with lower FA. ${ }^{50}$ It is possible that altered white matter connectivity is compensatory to effects from the illness on white matter integrity. In our previous study in anorexia nervosa after recovery, we found that longer duration of illness predicted greater connectivity strength. ${ }^{30}$ In this study we found some indication for a similar relationship, but less strongly not surviving multiple comparison correction. This could be due to the fact that subjects were still in the ill phase, and with ongoing illness this relationship may strengthen. We are currently recruiting subjects from this cohort when recovered in order to shed further light on this question.

To the best of our knowledge, this is the first study that provides evidence that structural white matter connectivity is related to individual sweet-taste perception. In all three groups, connectivity between middle OFC and insula was positively correlated with subjective sweetness perception, although this was found on the right in controls and in the eating-disorder groups on the left. The mechanistic underpinnings for such a relationship are elusive and require further study. In addition, in anorexia nervosa, structural connectivity correlated negatively with sweet perception between the ventromedial posterior nucleus of the thalamus and the insula, as well as fiber paths originating from the left amygdala and connections between left posterior insula and gyrus rectus. The mean connection strength 
between anorexia nervosa and controls between those regions was not different, and the implications of this negative correlation are speculative.

The effective conectivity, or dynamic causal mechanisms of brain circuits' interaction during tasting sucrose solution showed similarities but also fundamentally different patterns between groups. All groups showed bilaterally effective connectivity from insula regions to ventral striatum and OFC, as well as prefrontalOFC connectivity on the right side. We are not aware that this has been studied previously in this manner in humans. Taste information is thought to be transmitted via the thalamus to insula/frontal operculum and from there to anterior cingulate, ventral striatum, OFC and hypothalamus; ${ }^{51}$ however, new research using salient stimuli suggests a greater complexity of those pathways. ${ }^{52}$ One study suggested effective connectivity based on attention to monosodium glutamate taste intensity (PFC to insula) versus pleasantness (PFC to OFC).$^{53}$ Our data now provide new information how sweet taste may activate the taste-reward system. In controls, bilaterally, the flux of activation was directed from the hypothalamus to ventral striatum, suggesting that hypothalamic signals have an important input on ventral striatal activation and maybe motivation to approach food stimuli. In contrast, in both anorexia and bulimia nervosa the right anterior cingulate-effective connectivity was directed to the ventral striatum, which in turn mediated hypothalamus activity. This reversal of input may have key effects on appetite regulation in eating disorders. Basic science suggests that hypothalamusventral striatum connections are important for feeding regulation. ${ }^{54}$ Our data suggest that in eating disorders there may be a top-down control of this circuitry. The anterior cingulate is important in error-monitoring and anxiety-processing, ${ }^{55}$ and eating-related fearful cognitions could have an impact on subcortical taste-reward processing, which in turn could alter the normal hypothalamic feeding-drive input.

In anorexia nervosa, bilaterally effective connectivity was directed from frontal operculum to the anterior cingulate, and bulimia nervosa showed anterior cingulate-effective connectivity to the medial OFC. Input from the frontal operculum to the anterior cingulate could reinforce food avoidance behavior and anterior cingulate-OFC-effective connectivity could have an impact on value computation of sweet taste perception or hedonic experience in bulimia nervosa; however, testing this hypothesis will require a specific study design..$^{53}$

We did not see overlap between areas of altered groupeffective connectivity and structural connectivity strength. This suggests that those results and potential function-behavior implications are independent. On the other hand, altered white matter connectivity strength could be compensatory and normalize effective dynamic connectivity. White matter integrity was lower in most pathways tested in the anorexia but only in few white matter tracts in bulimia nervosa compared with controls. One interpretation is that FA could be related to the severity of malnutrition. This will need further study and across time from illness through the recovery process.

\section{Limitations}

The sample size was modest and requires replication. However, partial eta squared for connectivity strength between group comparisons was $>0.5$, indicating large effect size. Probabilistic tractography does not provide absolute fiber counts. ${ }^{24}$ Still, this method does provide results comparable to direct white matter neuron-tracing, ${ }^{56}$ suggesting that our results are valid. Utilizing 25 diffusion directions during MRI data acquisition may limit probabilistic tractography analysis; however, it has been shown that increasing diffusion directions does not improve fibertracking. ${ }^{57}$ The mechanism of greater white matter connectivity in eating disorders is uncertain, but could be due to white matter reorganization after tissue injury. ${ }^{50}$ Future studies will need to further explore whether alterations found result from underweight or are premorbid traits. The IMaGES algorithm used here may be one of the most reliable tools available, ${ }^{42}$ but it cannot describe whether the effective connectivity is increasing or decreasing activity between connected regions. The effective connectivity analysis found across-group connectivity in the same or opposite direction, or effective connectivity between regions in one group but not in another, and a limitation of the method is that direct comparison of connectivity parameters across groups cannot reliably be done based on those connectivity patterns. A limitation of this study is the cross-sectional design, and we are currently studying individuals with anorexia nervosa during recovery and hope that this will help better describe potential underlying mechanisms. This is a typical clinical eating-disorder sample with typical comorbidity of anxiety and depressive disorders. In order to control for those effects we included comorbidity (anxiety disorder and major depressive disorder) and medication use (selective serotonin reuptake inhibitor and antipsychotic) in the statistical model as factors but we cannot entirely rule out the impact of the comorbid conditions on the results. Anxiety and depression ratings did not significantly correlate with connectivity measures. In order to understand the mechanisms of connectivity strength as well as effective connectivity, it will be important to study those variables at different time points during disease and recovery. Ideally, a longitudinal approach is chosen. One very recent study exists that investigated effective connectivity and connectivity strength before and after weight restoration. That study included only one fiber path, the connection from the nucleus accumbens to the OFC, which was increased at both time points. ${ }^{29}$ Effective connectivity is an up to date less commonly used technique. We still are just at the beginning of understanding how brain regions interact, and we cannot exclude that effects from comorbid conditions had an impact on the effective connectivity results. In depression, for instance, a study found greater connectivity within the anterior cingulate cortex during a cognitive task compared with controls. ${ }^{58}$ Another study reported on lower prefrontal cortical-amygdala-effective connectivity in response to negative emotional faces in women with postpartum depression. In addition, a study in youth indicated that adolescents with major depressive disorder had lower effective connectivity from the amygdala to the anterior cingulate. ${ }^{59}$ In a study in social anxiety disorder the individuals with anxiety showed connectivity from the OFC to the amygdala, which was not observed in controls. ${ }^{60}$ Because those studies did not use taste stimuli or focused on, for instance, hypothalamus circuitry they are not comparable with this study. However, a study in controls showed that taste stimulation was related to effective connectivity from the insular cortex to the thalamus, a direction that we observed in our study subjects as well. ${ }^{61}$

\section{CONCLUSION}

This study suggests greater white matter connection strength across frontostriatal reward pathways, but reduced connectivity strength to the hypothalamus, which could have important implications on appetite regulation. The effective network connectivity from anterior cingulate to ventral striatum and to the hypothalamus in eating disorders provides a possible biological correlate for the hypothesis that those individuals are able to override homeostatic signals.

\section{CONFLICT OF INTEREST}

The authors declare no conflict of interest. 


\section{ACKNOWLEDGMENTS}

NIMH grants K23 MH080135, R01 MH096777 and R01 MH103436 provided funding for all aspects of the study to Dr Frank. We are grateful to all the individuals who participated in this study.

\section{REFERENCES}

1 Crow SJ, Peterson CB, Swanson SA, Raymond NC, Specker S, Eckert ED et al. Increased mortality in bulimia nervosa and other eating disorders. Am J Psychiatry 2009; 166: 1342-1346.

2 Becker AE, Grinspoon SK, Klibanski A, Herzog DB. Eating disorders. N Engl J Med 1999; 340: 1092-1098.

3 Halmi KA, Sunday SR. Temporal patterns of hunger and fullness ratings and related cognitions in anorexia and bulimia. Appetite 1991; 16: 219-237.

4 Strober M, Freeman R, Lampert C, Diamond J, Kaye W. Controlled family study of anorexia nervosa and bulimia nervosa: evidence of shared liability and transmission of partial syndromes. Am J Psychiatry 2000; 157: 393-401.

5 Volkow ND, Wang GJ, Baler RD. Reward, dopamine and the control of food intake: implications for obesity. Trends Cogn Sci 2011; 15: 37-46.

6 Gerard N, Pieters G, Goffin K, Bormans G, Van Laere K. Brain type 1 cannabinoid receptor availability in patients with anorexia and bulimia nervosa. Biol Psychiatry 2011; 70: 777-784.

7 Kaye WH, Wierenga CE, Bailer UF, Simmons AN, Wagner A, Bischoff-Grethe A. Does a shared neurobiology for foods and drugs of abuse contribute to extremes of food ingestion in anorexia and bulimia nervosa? Biol Psychiatry 2013; 73: 836-842.

8 Yoshida R, Niki M, Jyotaki M, Sanematsu K, Shigemura N, Ninomiya Y. Modulation of sweet responses of taste receptor cells. Semin Cell Dev Biol 2013; 24: 226-231.

9 Corwin RL, Avena NM, Boggiano MM. Feeding and reward: perspectives from three rat models of binge eating. Physiol Behav 2011; 104: 87-97.

10 Manuel-Apolinar L, Rocha L, Damasio L, Tesoro-Cruz E, Zarate A. Role of prenatal undernutrition in the expression of serotonin, dopamine and leptin receptors in adult mice: implications of food intake. Mol Med Rep 2014; 9: 407-412.

11 Frank GK, Shott ME, Hagman JO, Mittal VA. Alterations in brain structures related to taste reward circuitry in ill and recovered anorexia nervosa and in bulimia nervosa. Am J Psychiatry 2013; 170: 1152-1160.

12 Rolls B, Rolls E, Rowe E, Sweeney K. Sensory specific satiety in man. Physiol Behav 1981; 27: 137-142.

13 Amianto F, Caroppo P, D'Agata F, Spalatro A, Lavagnino L, Caglio M et al. Brain volumetric abnormalities in patients with anorexia and bulimia nervosa: a voxelbased morphometry study. Psychiatry Res 2013; 213: 210-216.

$14 \mathrm{Kim} \mathrm{KR}, \mathrm{Ku}$ J, Lee JH, Lee $\mathrm{H}$, Jung YC. Functional and effective connectivity of anterior insula in anorexia nervosa and bulimia nervosa. Neurosci Lett 2012; 521: 152-157.

15 Lee S, Ran Kim K, Ku J, Lee JH, Namkoong K, Jung YC. Resting-state synchrony between anterior cingulate cortex and precuneus relates to body shape concern in anorexia nervosa and bulimia nervosa. Psychiatry Res 2014; 221: 43-48.

16 Brooks SJ, O'Daly OG, Uher R, Friederich HC, Giampietro V, Brammer M et al. Differential neural responses to food images in women with bulimia versus anorexia nervosa. PLoS One 2011; 6: e22259.

17 Frank GK, Reynolds JR, Shott ME, Jappe L, Yang TT, Tregellas JR et al. Anorexia nervosa and obesity are associated with opposite brain reward response. Neuropsychopharmacology 2012; 37: 2031-2046.

18 Frank GK, Reynolds JR, Shott ME, O'Reilly RC. Altered temporal difference learning in bulimia nervosa. Biol Psychiatry 2011; 70: 728-735.

19 Oberndorfer TA, Frank GK, Simmons AN, Wagner A, McCurdy D, Fudge JL et al. Altered insula response to sweet taste processing after recovery from anorexia and bulimia nervosa. Am J Psychiatry 2013; 170: 1143-1151.

20 Kazlouski D, Rollin MD, Tregellas J, Shott ME, Jappe LM, Hagman JO et al. Altered fimbria-fornix white matter integrity in anorexia nervosa predicts harm avoidance. Psychiatry Res 2011; 192: 109-116.

21 Mettler LN, Shott ME, Pryor T, Yang TT, Frank GK. White matter integrity is reduced in bulimia nervosa. Int J Eat Disord 2013; 46: 264-273.

22 Via E, Zalesky A, Sanchez I, Forcano L, Harrison BJ, Pujol J et al. Disruption of brain white matter microstructure in women with anorexia nervosa. J Psychiatry Neurosci 2014; 39: 130135.

23 Diffusion Tractography (http://www.humanconnectome.org/about/project/tracto graphy.html). Accessed on 2015.

24 Jones DK, Knosche TR, Turner R. White matter integrity, fiber count, and other fallacies: the do's and don'ts of diffusion MRI. Neuroimage 2013; 73: 239-254.

25 Ramsey JD, Hanson SJ, Glymour C. Multi-subject search correctly identifies causal connections and most causal directions in the DCM models of the Smith et al. simulation study. Neuroimage $2011 ;$ 58: 838-848.
26 Perez C, El-Sheikh E, Glymour C. Discovering effective connectivity among brain regions from functional MRI data. Int J Comput Healthcare 2010; 1 : 86-101.

27 Kullmann S, Giel KE, Teufel M, Thiel A, Zipfel S, Preissl H. Aberrant network integrity of the inferior frontal cortex in women with anorexia nervosa. Neuroimage Clin 2014; 4: 615-622.

28 Suchan B, Bauser DS, Busch M, Schulte D, Gronemeyer D, Herpertz S et al. Reduced connectivity between the left fusiform body area and the extrastriate body area in anorexia nervosa is associated with body image distortion. Behav Brain Res 2013; 241: 80-85.

29 Cha J, Ide JS, Bowman FD, Simpson HB, Posner J, Steinglass JE. Abnormal reward circuitry in anorexia nervosa: a longitudinal, multimodal MRI study. Hum Brain Mapp 2016; advance online publication, 8 June 2016; doi: 10.1002/hbm.23279 (e-pub ahead of print).

30 Shott ME, Pryor TL, Yang TT, Frank GK. Greater insula white matter fiber connectivity in women recovered from anorexia nervosa. Neuropsychopharmacology 2016; 41: 498-507.

31 Wierenga CE, Ely A, Bischoff-Grethe A, Bailer UF, Simmons AN, Kaye WH. Are extremes of consumption in eating disorders related to an altered balance between reward and inhibition? Front Behav Neurosci 2014; 8: 410.

32 Torsello A, Brambilla F, Tamiazzo L, Bulgarelli I, Rapetti D, Bresciani E et al. Central dysregulations in the control of energy homeostasis and endocrine alterations in anorexia and bulimia nervosa. J Endocrinol Invest 2007; 30: 962-976.

33 Garner D. Eating Disorder Inventory ${ }^{\mathrm{TM}}-3$ (ED/TM-3). Psychological Assessment Resources, Inc.: Lutz, FL, USA, 2004.

34 Cloninger C, Przybeck T, Svrakic D, Wetzel R. The Temperament and Character Inventory (TCl): A guide to its development and use.1994; Vol. 2, Chapter 4, pp 19-28.

35 Spielberger CD. Manual for the State-Trate Anxiety Inventory. Consulting Psychologists Press Inc.: Palo Alto, CA, USA, 1983.

36 Beck AT, Steer RA, Ball R, Ranieri W. Comparison of Beck Depression Inventories IA and -II in psychiatric outpatients. J Pers Assess 1996; 67: 588-597.

37 O'Connor R, Colder C, Hawk L. Confirmatory factor analysis of the Sensitivity to Punishment and Sensitivity to Reward Questionnaire. Personal Indiv Differ 2004; 37: 985-1002.

38 O'Doherty JP, Dayan P, Friston K, Critchley H, Dolan RJ. Temporal difference models and reward-related learning in the human brain. Neuron 2003; 38: 329-337.

39 Eickhoff SB, Jbabdi S, Caspers S, Laird AR, Fox PT, Zilles K et al. Anatomical and functional connectivity of cytoarchitectonic areas within the human parietal operculum. J Neurosci 2010; 30: 6409-6421.

40 Rolls ET. Taste, olfactory and food texture reward processing in the brain and obesity. Int J Obes 2011; 35: 550-561.

41 Tzourio-Mazoyer N, Landeau B, Papathanassiou D, Crivello F, Etard O, Delcroix N et al. Automated anatomical labeling of activations in SPM using a macroscopic anatomical parcellation of the MNI MRI single-subject brain. Neuroimage 2002; 15 : 273-289.

42 Ramsey JD, Hanson SJ, Hanson C, Halchenko YO, Poldrack RA, Glymour C. Six problems for causal inference from fMRI. Neuroimage 2010; 49: 1545-1558.

43 Benjamini $Y$, Hochberg $Y$. Controlling the false discovery rate: a practical and powerful approach to multiple testing. J R Statist Soc B 1995; 57: 289-300.

44 Ahima RS, Antwi DA. Brain regulation of appetite and satiety. Endocrinol Metab Clin North Am 2008; 37: 811-823.

45 Holland PC, Petrovich GD. A neural systems analysis of the potentiation of feeding by conditioned stimuli. Physiol Behav 2005; 86: 747-761.

46 Institute of Medicine (US) Committee on Dietary Guidelines Implementation. Determinants of food choice and prospects for modifying food attitudes and behavior. In: Thomas P (ed). Improving America's Diet and Health: From Recommendations to Action. National Academies Press: Washington, DC, USA, 1991.

47 Yarmolinsky DA, Zuker CS, Ryba NJ. Common sense about taste: from mammals to insects. Cell 2009; 139: 234-244.

48 Guarda AS, Schreyer CC, Boersma GJ, Tamashiro KL, Moran TH. Anorexia nervosa as a motivated behavior: relevance of anxiety, stress, fear and learning. Physiol Behav 2015; 152: 466-472.

49 Castro DC, Cole SL, Berridge KC. Lateral hypothalamus, nucleus accumbens, and ventral pallidum roles in eating and hunger: interactions between homeostatic and reward circuitry. Front Syst Neurosci 2015; 9: 90.

50 Wang Z, Xia M, Dai Z, Liang X, Song H, He Y et al. Differentially disrupted functional connectivity of the subregions of the inferior parietal lobule in Alzheimer's disease. Brain Struct Funct 2015; 220: 745-762.

51 Rolls ET. The affective and cognitive processing of touch, oral texture, and temperature in the brain. Neurosci Biobehav Rev 2010; 34: 237-245.

52 Ray S, Haney M, Hanson C, Biswal B, Hanson SJ. Modeling causal relationship between brain regions within the drug-cue processing network in chronic cocaine smokers. Neuropsychopharmacology 2015; 40: 2960-2968. 
53 Luo Q, Ge T, Grabenhorst F, Feng J, Rolls ET. Attention-dependent modulation of cortical taste circuits revealed by Granger causality with signal-dependent noise. PLoS Comput Biol 2013; 9: e1003265.

54 Stratford TR, Kelley AE. Evidence of a functional relationship between the nucleus accumbens shell and lateral hypothalamus subserving the control of feeding behavior. J Neurosci 1999; 19: 11040-11048.

55 Etkin A, Egner T, Kalisch R. Emotional processing in anterior cingulate and medial prefrontal cortex. Trends Cogn Sci 2011; 15: 85-93.

56 Gao Y, Choe AS, Stepniewska I, Li X, Avison MJ, Anderson AW. Validation of DTI tractography-based measures of primary motor area connectivity in the squirrel monkey brain. PLoS One 2013; 8: e75065.

57 Berman Jl, Lanza MR, Blaskey L, Edgar JC, Roberts TP. High angular resolution diffusion imaging probabilistic tractography of the auditory radiation. AJNR Am J Neuroradiol 2013; 34: 1573-1578.

58 Schlosser RG, Wagner G, Koch K, Dahnke R, Reichenbach JR, Sauer H. Frontocingulate effective connectivity in major depression: a study with fMRI and dynamic causal modeling. Neuroimage 2008; 43: 645-655.

59 Musgrove DR, Eberly LE, Klimes-Dougan B, Basgoze Z, Thomas KM, Mueller BA et al. Impaired bottom-up effective connectivity between amygdala and subgenual anterior cingulate cortex in unmedicated adolescents with major depression: results from a dynamic causal modeling analysis. Brain Connect 2015; 5: 608-619.
60 Sladky R, Hoflich A, Kublbock M, Kraus C, Baldinger P, Moser E et al. Disrupted effective connectivity between the amygdala and orbitofrontal cortex in social anxiety disorder during emotion discrimination revealed by dynamic causal modeling for FMRI. Cereb Cortex 2015; 25: 895-903.

61 Yeung AW, Tanabe HC, Suen JL, Goto TK. Taste intensity modulates effective connectivity from the insular cortex to the thalamus in humans. Neuroimage 2016; 135: 214-222.

62 American Psychiatric Association. Diagnostic and Statistical Manual of Mental Disorders - Text Revision (DSM-IV-TR). Handbook of Psychiatric Measures. 4th edn, American Psychiatric Association: Washington DC, USA, 2000.

\section{c) (i)}

This work is licensed under a Creative Commons Attribution 4.0 International License. The images or other third party material in this article are included in the article's Creative Commons license, unless indicated otherwise in the credit line; if the material is not included under the Creative Commons license, users will need to obtain permission from the license holder to reproduce the material. To view a copy of this license, visit http://creativecommons.org/licenses/ by/4.0/

(c) The Author(s) 2016

Supplementary Information accompanies the paper on the Translational Psychiatry website (http://www.nature.com/tp) 